\title{
Dynamics of the Potato Root (Solanum Spp.) Under Different Levels of Soil Moisture, in the Geographical Region of Riobamba, Ecuador
}

\section{Dinámica Radicular de la Papa (Solanum Spp.) Bajo Diferentes Niveles de Humedad del Suelo, en el Entorno Geográfico de Riobamba- Ecuador}

VII International Congress of

Science, Technology,

Entrepreneurship and

Innovation (SECTEI 2020)

Corresponding Author:

R, Peña Murillo

robinson.pena@espoch.edu.ec

Published: 26 August 2021

Production and Hosting by Knowledge E

(c) R, Peña Murillo et al. This article is distributed under the terms of the Creative Commons Attribution License, which permits unrestricted use and redistribution provided that the original author and source are credited.
S OPEN ACCESS

\section{R, Peña Murillo, J. Velasteguí Cáceres, and J. León Ruiz}

Grupo de Investigación y Transferencia de Tecnología en Recursos Hídricos (GITRH), Centro Experimental del Riego (CER), Facultad de Recursos Naturales, Escuela Superior Politécnica de Chimborazo, Riobamba, Ecuador

\section{Abstract}

The root system of the potato (Solanum spp.) is often characterized as superficial and inefficient, with little capacity to extract water from the soil. Through a geographical study of the study area and root dynamics, the root behavior of the Victoria variety in Riobamba, Ecuador was evaluated, with the aim of assessing the applicability of "drip irrigation strategies with different levels of soil moisture" that allow the optimization of the water resource and the degree of tolerance of the crop to the lack of water through three treatments, $25 \%, 50 \%$ and $75 \%$, between the levels of field capacity and permanent wilting point that are equivalent to $12.25 \%$ (T1), $10.5 \%$ (T2) and $8.75 \%$ (T3) of sandy loam soil moisture. Through front-wall rhizotrons the gravimetric method for recording soil moisture, and the Scheffé test, with a randomized block experimental design, were completed. The maximum root development was reached 110 days after the cultivation was established, with $77.5 \mathrm{~cm}$ (T3) at lower water availability. The maximum yield was reached at 132 days, with $34.72 \mathrm{t} / \mathrm{ha}$ (T1), followed by $32.11 \mathrm{t} / \mathrm{ha}$ (T2) with 126 days, and $28.45 \mathrm{t} / \mathrm{ha}$ (T3) with 121 days. Therefore, it is concluded that the maximum permissible humidity level for the variety is $10.5 \%$, since lower levels would generate large losses.

Keywords: rizotron, drip irrigation, soil moisture, geographical analysis.

\section{Resumen}

El sistema de raíces de la papa (Solanum spp.) a menudo se caracteriza por ser superficial e ineficiente, con poca capacidad para extraer agua del suelo. Mediante un estudio geográfico del área de estudio y la dinámica radicular se evalúa el comportamiento radicular de la variedad victoria en Riobamba-Ecuador, con el objetivo de valorar la aplicabilidad de estrategias de "riego por goteo con diferentes niveles de humedad en el suelo" que permitan la optimización del recurso hídrico y el grado de tolerancia del cultivo a la falta de agua mediante tres tratamientos $25 \%, 50 \%$ y $75 \%$ entre los niveles de capacidad de campo y punto de marchitez permanente que equivalen a $12,25 \%(\mathrm{~T} 1), 10,5 \%(\mathrm{~T} 2)$ y $8,75 \%(\mathrm{~T} 3)$ de humedad del suelo franco arenoso. A través de rizotrones de pared frontal, el método gravimétrico para el registro de la humedad del suelo y la prueba de Scheffé, con un diseño experimental de bloques completos al azar. El máximo desarrollo radicular se alcanzó a los 110 días de establecido el cultivo con $77,5 \mathrm{~cm}$ (T3) a una menor disponibilidad de agua. El rendimiento máximo se alcanzó a los 132

How to cite this article: Murillo RP, Cáceres JV, Ruiz JL (2021). Dynamics of the Potato Root (Solanum Spp.) Under Different Levels of Soil Moisture, in the 
días con 34,72t/ha (T1), seguido de 32,11t/ha (T2) con 126 días y 28,45t/ha (T3) con 121 días. Por lo tanto, se concluye que el máximo nivel de humedad permisible para la variedad es $10,5 \%$, ya que niveles inferiores generarían grandes pérdidas.

Palabras Clave: rizotrón, riego por goteo, humedad del suelo, análisis geográfico.

\section{Introducción}

La papa (Solanum tuberosum L.) es uno de los principales cultivos tradicionales, orientado al consumo de la población, ocupando el cuarto lugar a nivel mundial, el mismo que se cultiva en 19 millones de hectáreas, con una producción anual de alrededor de 325 millones de toneladas [1, 2]. Mencionar que los países en desarrollo producen más de la mitad de la producción total mundial de papa [3] mismos que han expandido sus áreas de cultivo, sin considerar que en cierta medida esta expansión se vea afectado negativamente por el cambio climático global $[4,5]$.

La planta de papa es sensible a la sequía y por lo tanto, susceptible a las oscilaciones climáticas [6, 7]. Una respuesta de los cultivos al estrés hídrico o por nutrientes es cultivar más raíces para acceder a más recursos en el suelo [8]. Otra respuesta se puede ver en la plasticidad de la raíz, donde las raíces crecen hacia el agua o los nutrientes disponibles [9-11]. Por lo tanto, es necesario prácticas de riego eficientes que proporcionen un uso sostenible del agua en el futuro, mediante la dotación de láminas de riego exactas para la determinación del grado de tolerancia que presenta el cultivo a la falta de agua [8, 9].

Los ciclos de riego son cruciales para producir y mantener las señales relacionadas con el 'estrés-raíz' sin afectar el estado hídrico de la planta, con buenos rendimientos con un ahorro considerable y una mayor eficiencia en el uso del agua, siendo de suma importancia en las zonas donde los recursos hídricos son limitados [9, 12].

En consecuencia, no se ha profundizado en investigaciones sobre la evaluación del comportamiento de la raíz de papa en función a la disponibilidad de agua, con el propósito de alterar la fisiología de la planta y explotar eficientemente las señales hidráulicas y químicas para obtener beneficios agronómicos de interés comercial como pueden ser: controlar un excesivo vigor, incrementar la calidad del fruto, ahorrar y mejorar la eficiencia en el uso del agua $[13,14]$, por lo tanto se presenta con la investigación evaluar la dinámica radicular y comportamiento biofísico de la papa variedad victoria bajo diferentes niveles de humedad del suelo, además del uso del agua en cada uno de los niveles de humedad del suelo. 


\section{Metodología}

\subsection{Análisis Geográfico del Área de Estudio}

La presente investigación se realizó en los predios del Centro Experimental del Riego (CER) de la Facultad de Recursos Naturales de la Escuela Superior Politécnica de Chimborazo (ESPOCH), Cantón Riobamba, Provincia de Chimborazo, Ecuador, a una altitud de 2821 m.s.n.m. Latitud: 1³9'18.82' 'S, Longitud: 7840’39.99”'W, coordenadas: UTM 17S Datum WGS84, X:758232 Y:9817776. La temperatura media es de $13.5^{\circ} \mathrm{C}$, una precipitación media anual de $350 \mathrm{~mm} /$ ño y la humedad relativa de 59,6\%. Además, según Holdrige (1982), la localidad se encuentra en el rango de estepa espinosa Montano Bajo (eeMB). Los suelos del CER son de textura franco-arenosa, con una capacidad de campo (CC) de 14\%, punto de marchitez permanente (PMP) de $7 \%$, agua útil $7 \%$ y densidad aparente de $1,5 \mathrm{~g} / \mathrm{cm} 3$, el agua posee un $\mathrm{pH} 8,7$ alcalino, una conductividad eléctrica $940 \mu S$ y una turbidez alta 0,9.

Como materiales se utilizaron: semilla de papa certificada variedad victoria, lisímetros de drenaje para la obtención de la evapotranspiración, rizotrones de pared frontal para evaluar el crecimiento radicular, sistema de riego a goteo para la dotación de láminas exactas de riego, tanque evaporación clase $A$, estación meteorológica portátil para la evolución de temperatura y precipitación, GPS, cámara fotográfica, software (ARGIS, AutoCAD, SPSS, Office, Google Earth) e información cartográfica y pluviométrica.

Como uno de los requerimientos para el desarrollo del presente proyecto fue plasmar la ubicación del área de estudio en mapa a una escala 1:1000 como se observa en el Mapa 1, para lo cual se utilizó las coordenadas obtenidas en campo con GPS, ortofoto obtenida en vuelo con UAV. Los insumos antes mencionados fueron utilizados como base para la generación de feature class de los componentes geográficos que se encuentran en el área de estudio, para lo cual se utilizó el software ArcGIS mismo que cuenta con el respectivo licenciamiento educativo. En el Mapa 1 se puede visualizar los datos geográficos en formato vectorial tipo polígono (T1, T2, T3, R1, R2, R3) que se generaron utilizando los datos geoespaciales base, lo que permitió delimitar las parcelas del entorno geográfico a ser estudiado.

\subsection{Método}

La primera fase de la investigación consistió en la recopilación de información base de investigaciones efectuadas por el CER concernientes a la optimización del agua de riego y el comportamiento radicular [15], además de lineamientos de la FAO con la determinación de la evapotranspiración del cultivo [16]. La investigación se enfocó en determinar el nivel máximo de tolerancia del cultivo a la falta de agua a través de la 


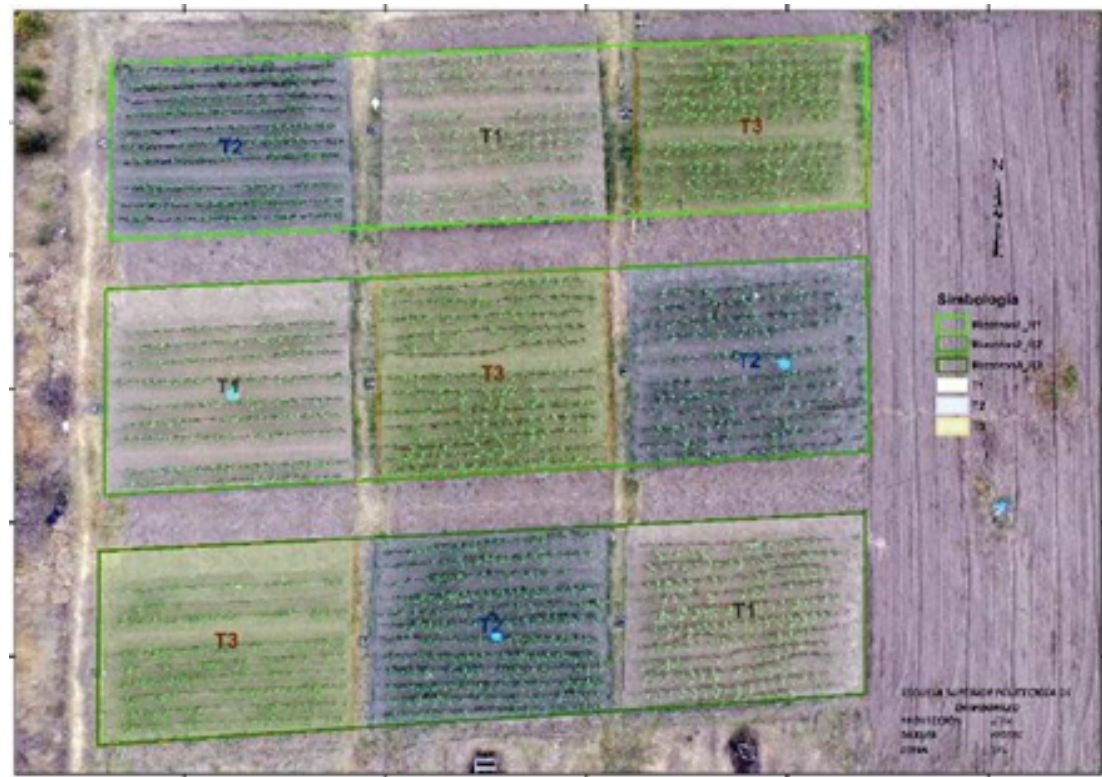

Figure 1

Delimitación del entorno geográfico con sus respectivos elementos de estudio.

restricción de la misma, tomando como antecedente que en la actualidad es complicado dotar el $100 \%$ de agua que demanda el cultivo debido a la disminución de la oferta hídrica, en este contexto se estableció tres tratamientos: $\mathrm{T} 1=25 \%$ del consumo de agua entre los niveles CC y PMP, T2 $=50 \%$ del consumo de agua entre los niveles CC y PMP, T3 $=75 \%$ del consumo de agua entre los niveles CC y PMP, mediante un diseño completamente al azar con tres repeticiones con un área por unidad experimental de $100 \mathrm{~m} 2$ y en si un área total de $1156 \mathrm{~m}^{2}$. Para los factores e interacciones con significancia estadística se utilizó la prueba de significación de Scheffé al 5\%.

Durante el ciclo de cultivo se llevó a cabo mediciones biométricas (altura de planta (4, 75 y 105 días), crecimiento radicular (45, 85 y 110 días) en base a los lineamientos de [17] para la variedad en estudio, además de la determinación del requerimiento hídrico. Se utilizó una estación meteorológica y un tanque estándar evaporímetro tipo A para evaluar el comportamiento climático (precipitación, temperatura y evaporación). En la cosecha se determinó rendimiento por categorías (comercial, semilla y no comercial) y rendimiento total [17].

\subsection{Variación de la Humedad del Suelo}

El porcentaje de humedad se determinó extrayendo una muestra de cada tratamiento a una profundidad de $0-30 \mathrm{~cm}$, en forma diaria y luego aplicando la ecuación $[14,18]$.

Contenido humedad suelo $(\%)=\left(\frac{P F-P S}{P S}\right) \times 100$ [19], dónde: PF Peso fresco de la muestra de suelo (g); PS Peso seco de la muestra de suelo (g). 


\subsection{Medición de la Evapotranspiración para los Tratamientos}

Para evaluar el efecto de los niveles de humedad del suelo se instaló un sistema de riego por goteo con una manguera auto compensadas con goteros cada $30 \mathrm{~cm}$, las mismas que se ubicó en cada lomo del surco, los riegos se efectuaron cuando se consumió el $25 \%$, 50\% y $75 \%$ entre los niveles de capacidad de campo (CC) y punto de marchitez (PMP), se presenta en la Tabla 1.

\section{Table 1}

Tratamientos en estudio.

$\begin{array}{ll}\text { Tratamien to } & \text { Descripción } \\ \text { T1 } & \begin{array}{l}\text { Variedad de papa Victoria con el } 25 \% \text { del consumo de agua entre los niveles de CC y } \\ \text { PMP. }\end{array} \\ \text { T2 } & \begin{array}{l}\text { Variedad de papa Victoria con el } 50 \% \text { del consumo de agua entre los niveles de CC y } \\ \text { PMP. }\end{array} \\ \text { T3 } & \begin{array}{l}\text { Variedad de papa Victoria con el } 75 \% \text { del consumo de agua entre los niveles de CC y } \\ \text { PMP. }\end{array}\end{array}$

Los lisímetros de drenaje se instalaron en cada tratamiento, para determinar la cantidad de agua a dotar en el siguiente riego es decir la lámina a reponer, aplicando la ecuación [20]:

$$
E t c=E t o * K c,[16]
$$

dónde: Etc: Evapotranspiración del cultivo $(\mathrm{mm})$; Eto: Evapotranspiración de cultivo de referencia $(\mathrm{mm}) ; \mathrm{Kc}$ : Coeficiente de cultivo La evapotranspiración del cultivo de referencia (Eto) es una cantidad que depende exclusivamente de las condiciones del medio ambiente misma se determinó con la ecuación [15]:

$$
E t o=E v * K p,[21]
$$

dónde: Ev: Evaporación (mm); Kp: Se calculó a partir de datos climáticos de la zona como humedad relativa y viento [14]: El coeficiente de cultivo Kc bajo estrés hídrico ajustado a cada tratamiento en estudio, en función al valor real obtenido con el lisímetro de drenaje se obtuvo a partir de la ecuación de Hargraves:

$$
K c=0.01335+0.04099 *(C)-0.0004 *(C) \wedge 2,[22]
$$

dónde: C: Días de desarrollo de cultivo (\%)

\subsection{Parámetros Biofísicos}

En función a la guía de toma de datos [17], la altura se midió a partir del cuello hasta el ápice del tallo en diez plantas elegidas al azar de la parcela neta, a los 45, 75 y 105 días después de la siembra [17]. 
La profundidad radicular se midió a partir del cuello de planta hasta la raíz con mayor profundidad, en dos plantas de la parcela neta a los 45, 85 y 110 días después de la siembra con lineamiento de [17] para la variedad victoria.

\subsection{Rendimiento}

El rendimiento por tamaño del tubérculo se clasificó los tubérculos cosechados de la parcela total en tres categorías: papa comercial de primera (peso mayor a $60 \mathrm{~g}$ ), papa para 'semilla' de segunda (peso entre 30 a $60 \mathrm{~g}$ ) y papa de desecho (peso menor a 30 g). Los resultados se expresarán en t/ha/categoría.

El rendimiento total se determinó cosechando la parcela neta de las tres repeticiones, la misma que se sumó y se sacó un promedio, el resultado se expresó en t/ha [17].

\section{Resultados y Discusión}

\subsection{Variación Climática durante la Fase Experimental}

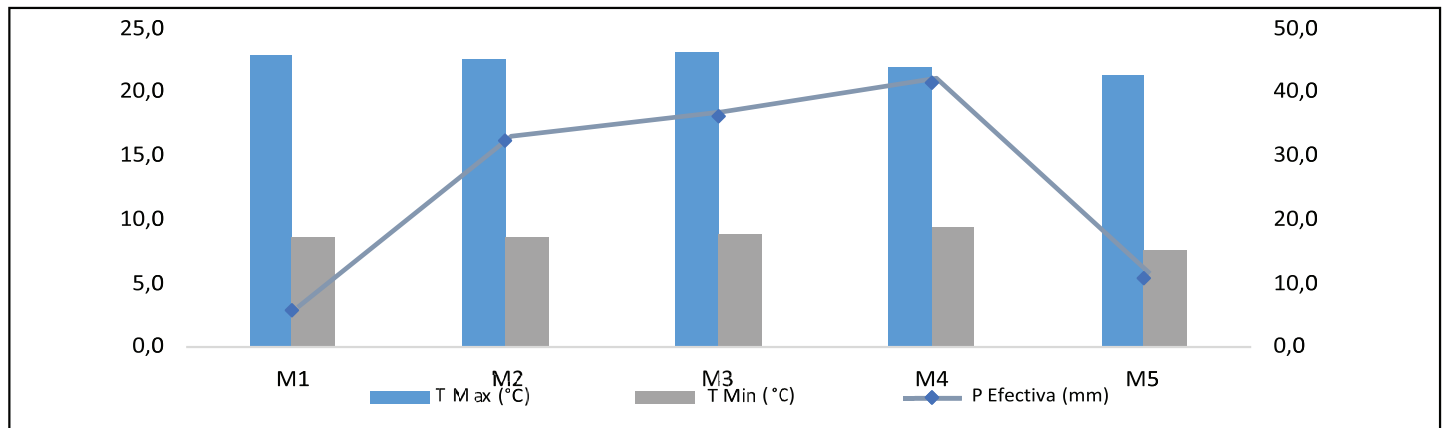

Figure 2

Promedios de temperatura $\left({ }^{\circ} \mathrm{C}\right)$ y precipitación efectiva $(\mathrm{mm})$ durante el ciclo de cultivo de papa (Solanum spp.) variedad victoria.

En la Figura 1 se observa una fluctuación no relevante de temperatura máxima registrando el mayor valor en el mes de noviembre con $23,1^{\circ} \mathrm{C}$, y en relación a la temperatura mínima esta presentó el valor mínimo en el mes de enero con $7,5^{\circ} \mathrm{C}$, cabe mencionar que durante este mes hubo registros de hasta $3^{\circ} \mathrm{C}$, lo que fue necesario tomar las medidas preventivas para evitar daños en el cultivo [23], el cultivo no tolera las temperaturas mínimas bajo los $10^{\circ} \mathrm{C}$ y máximas a $30^{\circ} \mathrm{C}$ mismas que inhabilitan el desarrollo del tubérculo. En referencia a la precipitación efectiva el máximo registrado es $41,4 \mathrm{~mm}$, lo que según [24], considera que precipitaciones inferiores a $10 \mathrm{~mm}$ no son efectivas para el cultivo ya que estas quedan retenidas en las hojas y se evaporan. 


\subsection{Variación de la Humedad del Suelo}

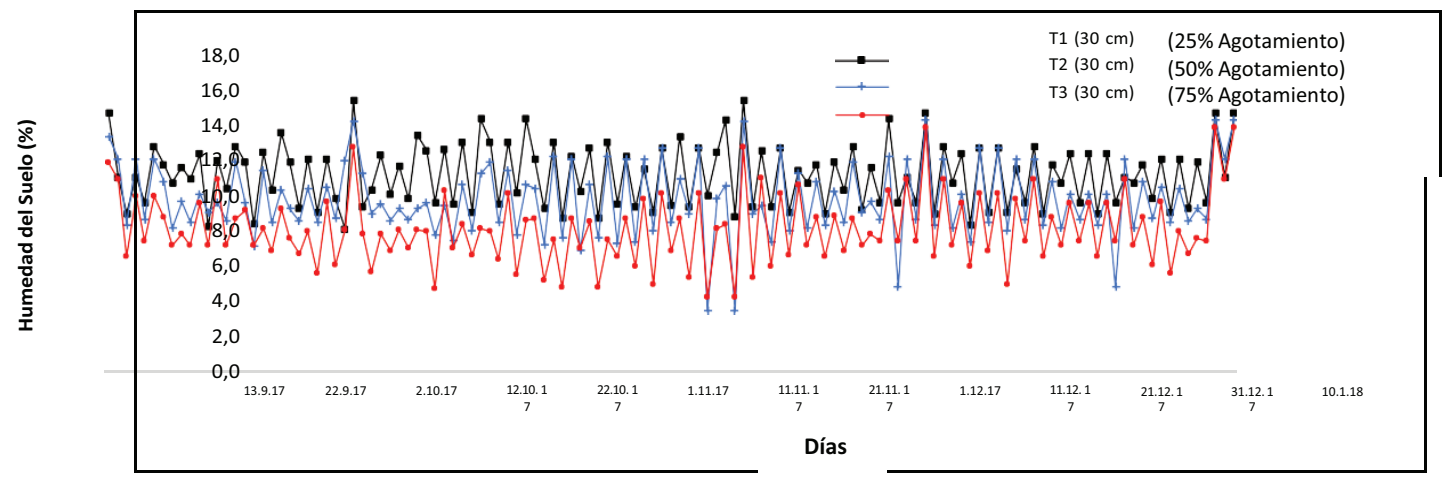

Figure 3

Registro de humedad del suelo a $30 \mathrm{~cm}$ de profundidad, durante el ciclo de cultivo de papa (Solanum spp.) variedad victoria.

En la Figura 2 se presenta el comportamiento de la humedad del suelo a la profundidad de $30 \mathrm{~cm}$, donde los patrones de humectación generan la configuración del sistema radicular, la planta en cada tratamiento se expuso a diferentes variaciones de humedad del suelo, donde el tratamiento 1 se mantuvo a un nivel de mayor de humedad con un promedio de $12,25 \%$, el tratamiento 2 con $10,5 \%$ y el tratamiento 3 con $8,75 \%$; además estos lapsos de agua ocasionan fisiológicamente un notorio comportamiento de la raíz [1,25], que esto influye en la conductividad hidráulica de la raíz a nivel celular, correlacionado con un elevado nivel de ácido abscísico (ABA).

\subsection{Altura de Planta}

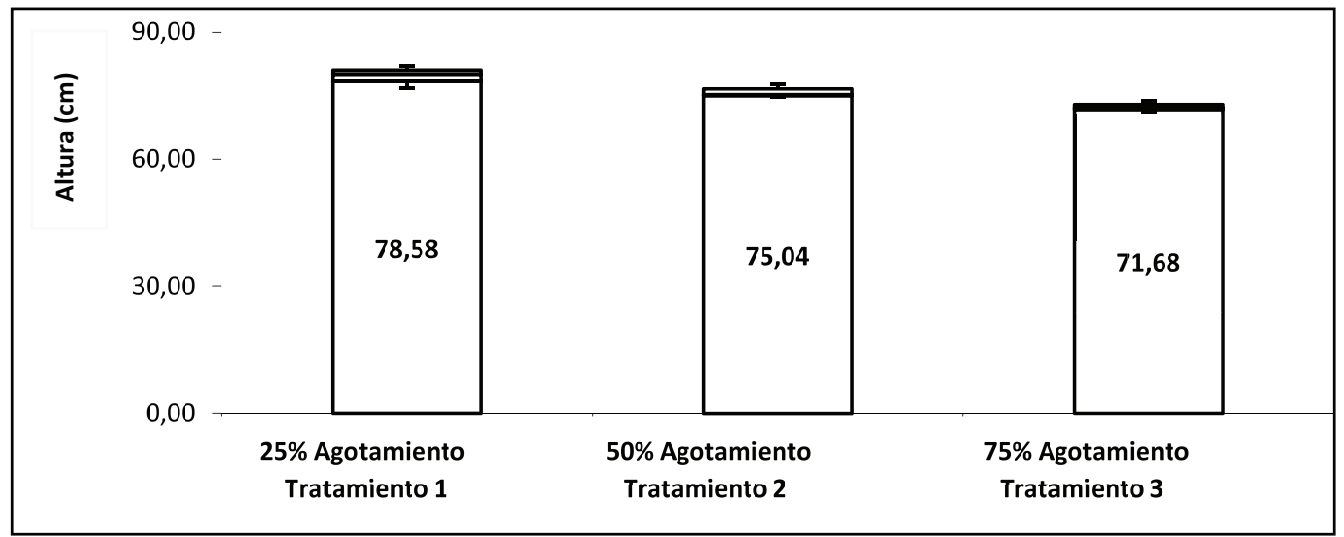

Figure 4

Promedios de altura (cm) a niveles de humedad del suelo en la papa (Solanum spp.) variedad victoria a los 105 días después de la siembra (dds). 
Al efectuar el análisis a los 45 días después de la siembra no presentó diferencias significativas entre tratamientos $(9,24$ ns) con un coeficiente de variación de $26,68 \%$. Referente a los 75 días después de la siembra, se presentó diferencias significativas entre tratamientos $\left(90,57^{*}\right)$, con un coeficiente de variación de $6,73 \%$ y así un promedio general de 49,74 cm para los tres tratamientos. A los 105 días después de la siembra se presentó diferencias significativas entre tratamientos $\left(35,75^{*}\right)$ ya que los diferentes niveles de humedad del suelo generaron respuestas notorias en la altura de la planta (Figura 3) con un coeficiente de variación de 3,02\% y un promedio general de 75,10 $\mathrm{cm}$, indicar con otras investigaciones [26], quienes consideran a la altura del tallo como un parámetro trascendental para evaluar la tolerancia a la falta de agua, reflejado en reprimir la división celular afectando así procesos fisiológicos y bioquímicos (fotosíntesis, translocación, metabolismo de nutrientes, entre otros) [2,10].

\subsection{Crecimiento de la Raíz a los 45 Días}

El análisis de varianza para crecimiento de la raíz a los 45 días después de la siembra señala diferencias no significativas entre tratamientos (104,65 ns), con un coeficiente de variación de $36,78 \%$ y un promedio general de $24,50 \mathrm{~cm}$. Se observó que a medida que crece la planta, las raíces reorganizan las partículas del suelo, en función a la compactación del suelo ya que es un factor condicionante que la planta tiene que vencer, lo que concuerda con $[4,27]$, que indican que la resistencia a la penetración del suelo influye en la tasa de elongación de la raíz y el diámetro de las raíces aumenta marcadamente, lo que lleva al crecimiento de raíces agrupadas y la extensión restringida de la raíz.

En los primeros estadios de desarrollo de la planta el comportamiento del sistema radicular (Figura 4), va a depender de la disponibilidad de agua en el suelo, como mencionan [12], el retener el agua de riego al principio de la temporada podría reducir la extensión de raíces de las papas en comparación con la situación de retener o reducir la cantidad de riego más adelante en la temporada, considerando que la papa tienen la capacidad deficiente para extraer agua y minerales del suelo $[28,29]$.

\subsection{Crecimiento de la Raíz a los 85 Días}

El análisis de varianza para crecimiento de la raíz a los 85 días después de la siembra señala diferencias significativas entre tratamientos $\left(277,65^{*}\right)$, con un coeficiente de variación $6,23 \%$ y un promedio general de $60,63 \mathrm{~cm}$, por lo tanto las variaciones en el contenido de agua del suelo ocasionaron una alteración del sistema radicular (Figura 5) donde las plantas mejoraron su capacidad de absorción de agua mediante la extensión del sistema de enraizamiento [30, 31]. La diferencia en profundidad es 


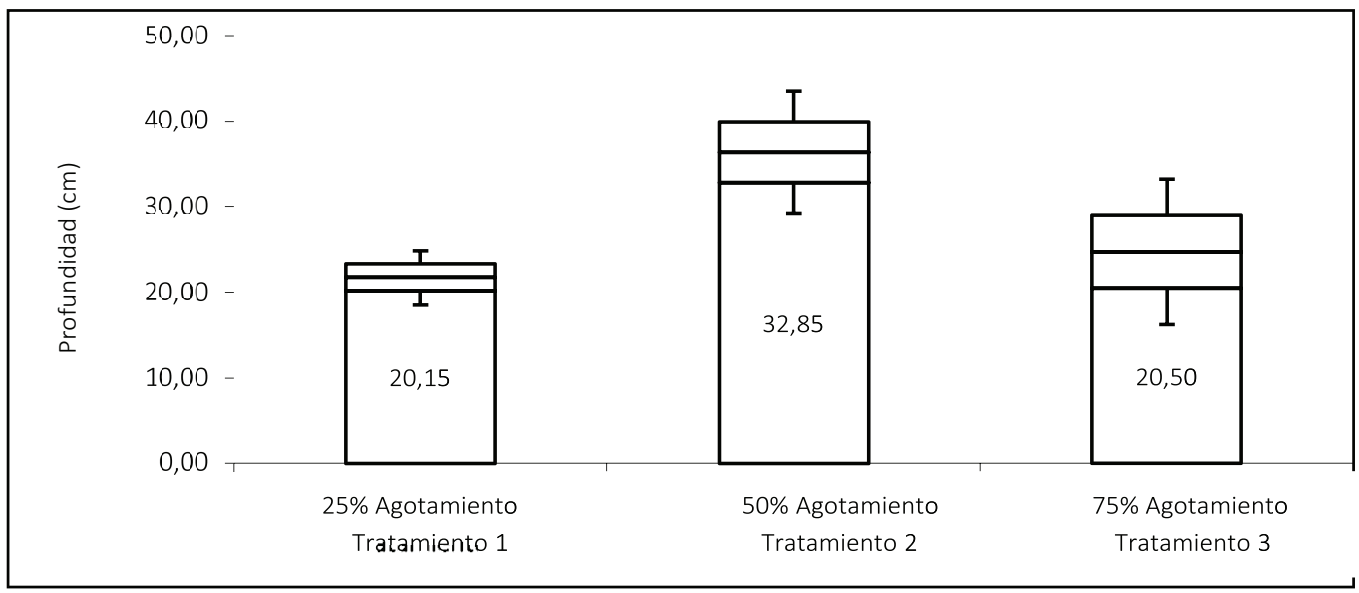

Figure 5

Promedios de raíz (cm) a niveles de humedad del suelo en la papa (Solanum spp.) variedad victoria a los 45 días después de la siembra (dds).

significativa, ubicando al T3 en el rango 'a' y T1 en el rango 'b' con una diferencia de $23,55 \mathrm{~cm}$. Considerar lo manifestado [32], el desarrollo de las raíces se reprime parcialmente con el inicio de la tuberización, lo que sugiere que las prácticas para mejorar el enraizamiento antes de esa etapa pueden contribuir a la eficacia de la raíz.

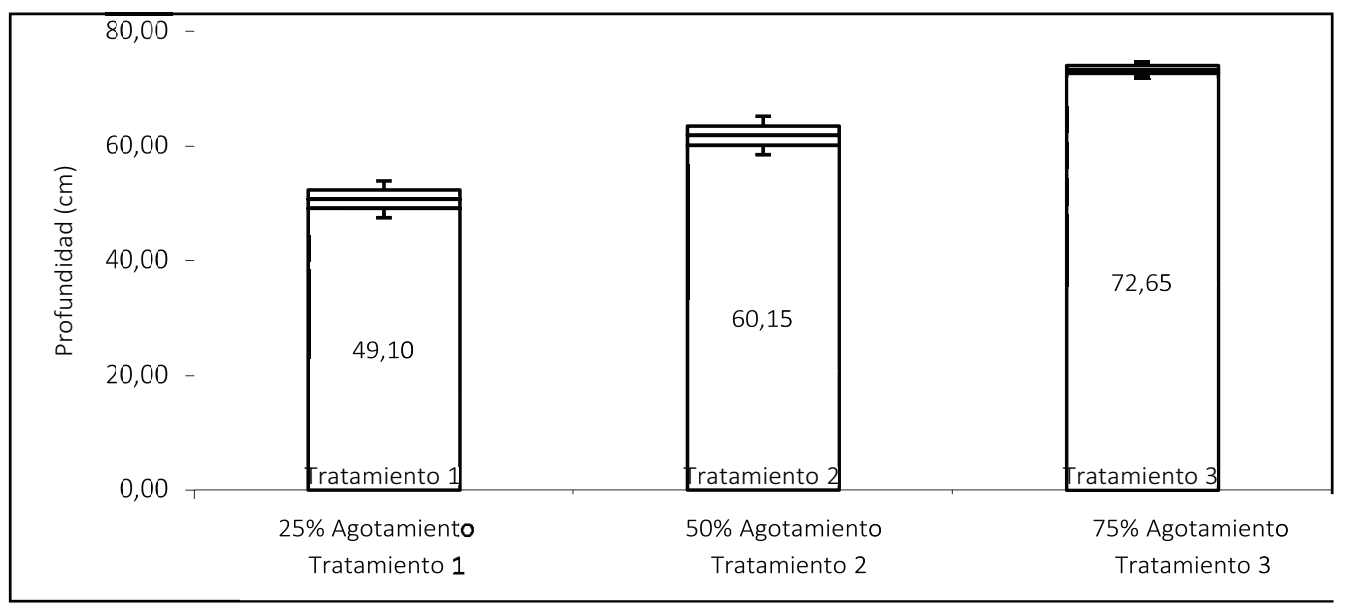

Figure 6

Promedios de raíz (cm) a niveles de humedad del suelo en la papa (Solanum spp.) variedad victoria a los 85 días después de la siembra (dds).

\subsection{Crecimiento de la Raíz a los 110 Días}

El análisis de varianza para crecimiento de la raíz a los 110 días después de la siembra señala diferencias significativas entre tratamientos $\left(173,05^{*}\right)$, con un coeficiente 
de variación $3,40 \%$ y un promedio general de $67,68 \mathrm{~cm}$, destacar los efectos de la disponibilidad de agua en el suelo en función al tiempo, hacen más notoria la respuesta la planta (Figura 6), si relacionamos con las investigaciones realizadas por [33], quienes demostraron que a pesar que la densidad de la raíz de la papa disminuye con la profundidad, estas raíces de las capas más profundas aportaban notablemente al requerimiento total de agua del cultivo, independiente del estado hídrico de las capas superiores del suelo, además que las raíces jóvenes de la capa profunda presentan una alta eficiencia de extracción de agua [12, 32]. Acotar que dicha eficiencia comparada con otros cultivos no es tan efectiva [15]. Por último, una posible explicación del aumento aparente en el crecimiento de la raíz en el suelo seco frente al suelo húmedo podría deberse a un menor contenido de oxígeno en el suelo húmedo [34, 35].

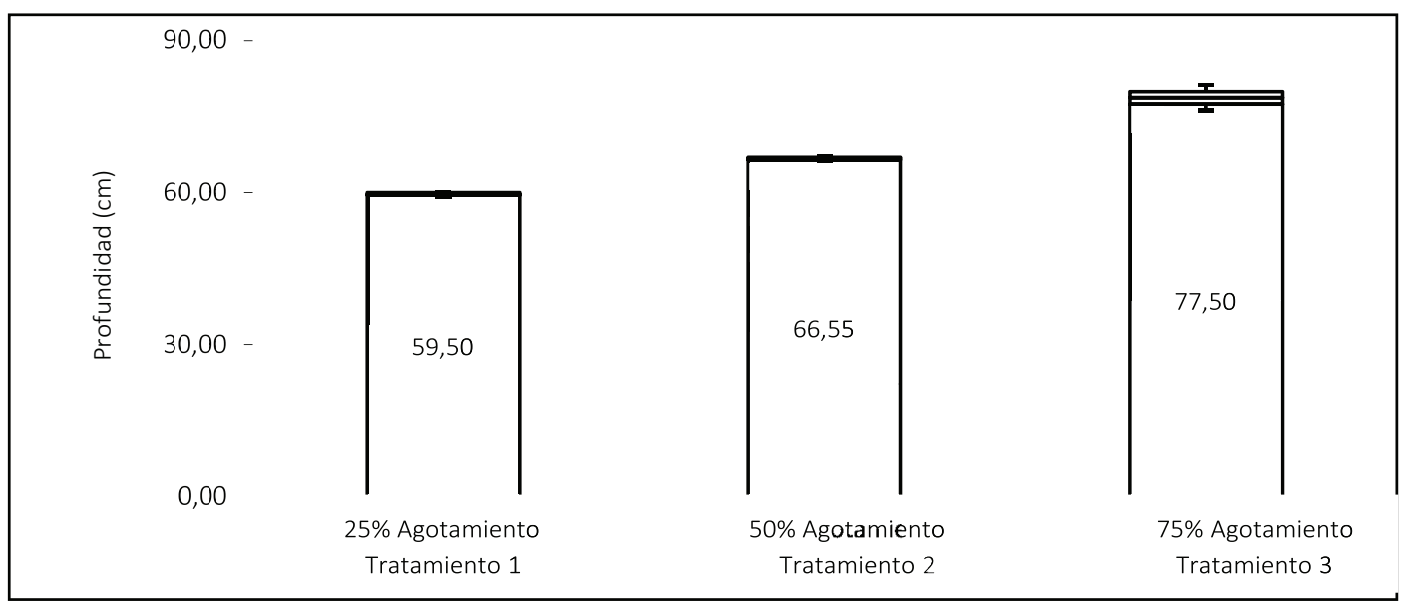

Figure 7

Promedios de raíz (cm) a niveles de humedad del suelo en la papa (Solanum spp.) variedad victoria a los 110 días después de la siembra (dds).

\subsection{Relación Bulbo de Humedecimiento y Sistema Radicular}

El comportamiento de la raíz (Figura 7), en los tres tratamientos presenta una mayor concentración de raíces hasta los primeros $30 \mathrm{~cm}$ desde el nivel del suelo, con un promedio de $63 \%$ y notoriamente la concentración desciende a mayor profundidad con un promedio de $37 \%$ esto se afirma en base a los estudios realizados por [ $21,36,37$, quienes señalan que el sistema de raíces de la papa a menudo se concentra en los 30 $\mathrm{cm}$ superiores de suelo y en cierta medida limita la accesibilidad de nutrientes, lo que genera que el cultivo sea más susceptible a la escasez de agua cuando se secan los suelos. Un aspecto importante que mencionar es que la profundidad de enraizamiento está controlada de manera efectiva por la curva característica de humedad del suelo, 


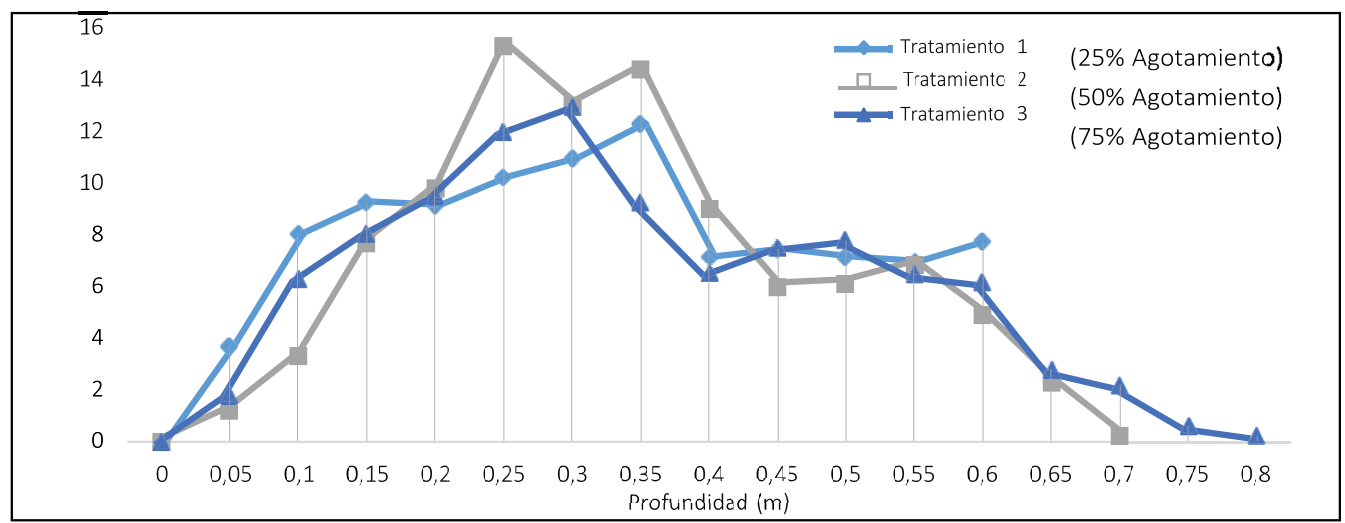

Figure 8

Comportamiento radicular en función al bulbo de humedad del suelo en la papa (Solanum spp.) variedad victoria.

en lugar de la densidad aparente. En tal sentido la distribución del agua en el suelo, la textura y estructura del suelo ejerce efectos sobre el crecimiento y la distribución de las raíces [11, 29, 38, 39]. Cabe mencionar que las limitaciones en la arquitectura de la raíz del cultivo a menudo limitan directamente su rendimiento [13, 39].

Al suministrar de forma alternada la dotación de agua en cada tratamiento este condiciona, el desarrollo radicular, correlacionado con varios estudios que mostraron que los sistemas de raíces de las plantas de riego parcial tenían una mayor capacidad de exploración del suelo más profunda y que la rehidratación del suelo seco mejoraba enormemente la asignación de biomasa a las raíces en ese suelo [40].

La profundidad de enraizamiento generalmente se extiende de $40 \mathrm{~cm}$ a $80 \mathrm{~cm}$ $[33,39,41]$, lo que en base a nuestros resultados podemos destacar lo siguiente:

En la Figura 8 el comportamiento radicular del tratamiento 1, llegó hasta los 60 $\mathrm{cm}$, debido a que la humedad del suelo se mantuvo en promedio de $12,25 \%$ lo que ocasionó la configuración del sistema radicular, además la mayor concentración de raíces se presentó en los primeros $30 \mathrm{~cm}$ con el 63,61\%.

En la Figura 9 se observa el tratamiento 2, en base al comportamiento de humedad del suelo que se mantuvo en promedio de 10,50\%, que ha influenciado a que el sistema radicular llegue a $70 \mathrm{~cm}$, con una tendencia a un mayor crecimiento de la raíz en la búsqueda de agua en capas más profundas del suelo, en comparación con el tratamiento 1.

En la Figura 10 el comportamiento radicular del tratamiento 3 fue mayor que los demás tratamientos ya que la planta tuvo que realizar un mayor esfuerzo en la búsqueda de agua, hasta alcanzar una profundidad de $80 \mathrm{~cm}$, esto debido a que el nivel de humedad del suelo se mantuvo en promedio 8,75\%. Además, la concentración de raíces en los últimos $40 \mathrm{~cm}$ fue de 40,18\%. 


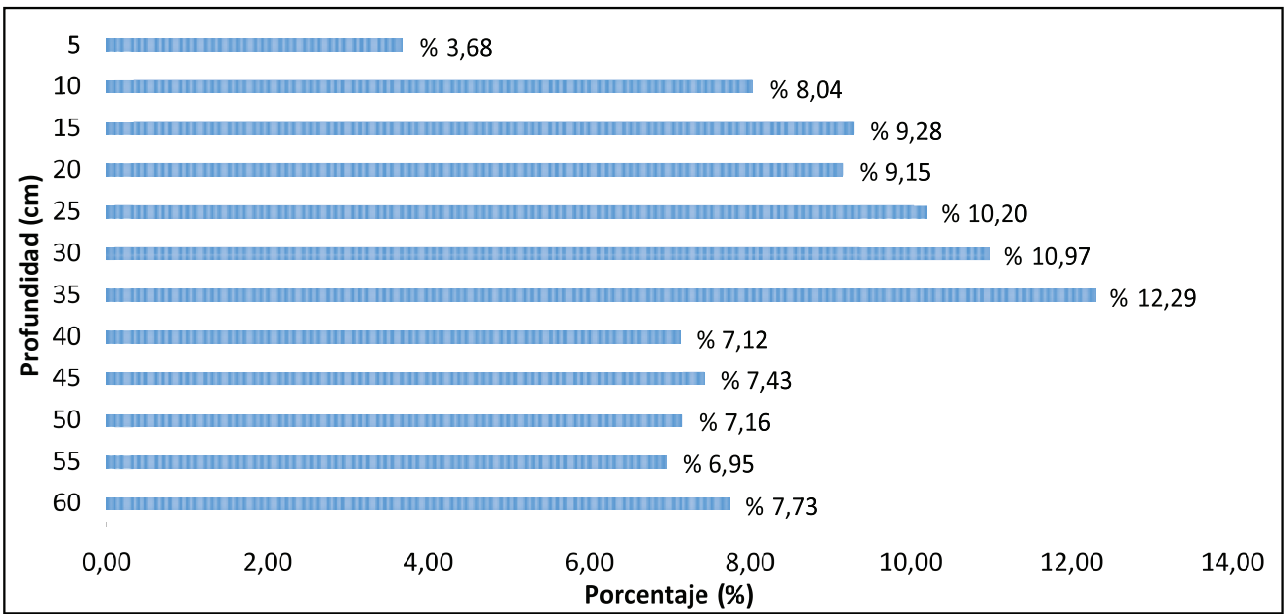

Figure 9

Patrón de extracción (\%) del tratamiento 1 (25\% agotamiento) a niveles de humedad del suelo en la papa (Solanum spp.) variedad victoria en función de la profundidad.

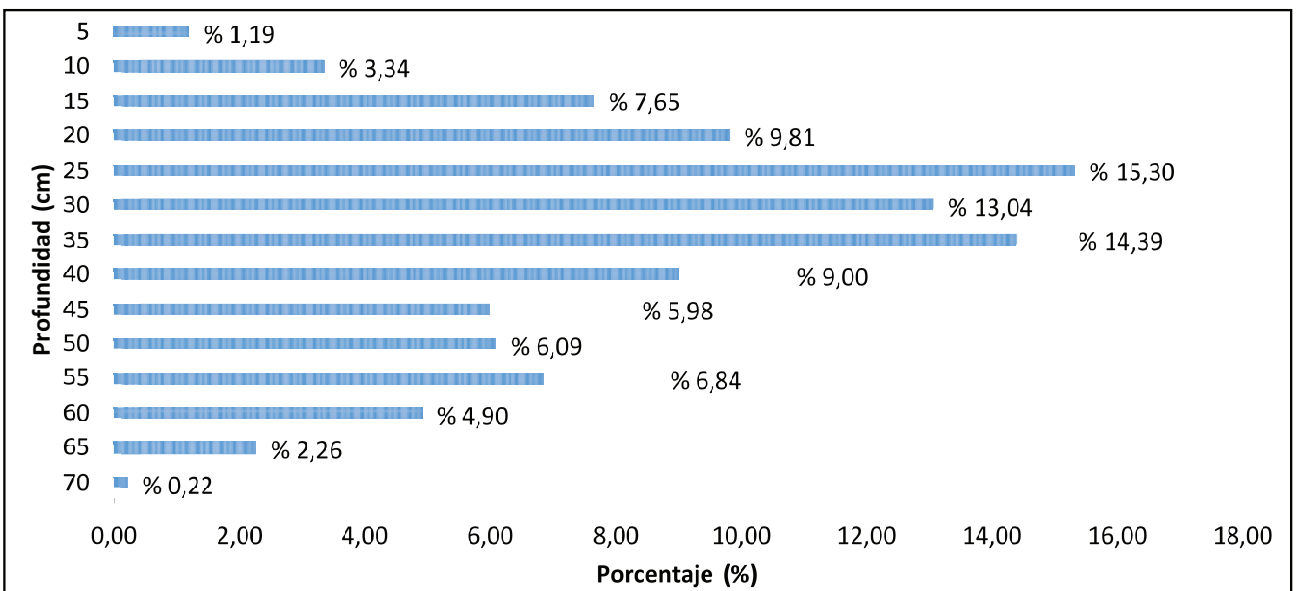

\section{Figure 10}

Patrón de extracción (\%) del tratamiento 2 (50\% agotamiento) a niveles de humedad del suelo en la papa (Solanum spp.) variedad victoria en función de la profundidad.

\subsection{Requerimiento Hídrico por Etapa Fenológica}

El requerimiento hídrico $(\mathrm{mm})$, durante el ciclo de cultivo de papa en cada uno de los tratamientos en estudio se presenta en la Tabla 2.

Durante el ciclo de cultivo, cada tratamiento presentó variaciones en función al grado de crecimiento vegetativo, profundidad radicular y fundamentalmente el rendimiento, influenciado por las condiciones medioambientales, características del suelo, las prácticas agrícolas y considerablemente las láminas de riego aplicadas [22, 23].

Por lo manifestado el requerimiento hídrico fue de $488.56 \mathrm{~mm}$ para el tratamiento 1; 455,28 mm para el tratamiento 2 y 428,43 $\mathrm{mm}$ para el tratamiento 3 resultados 


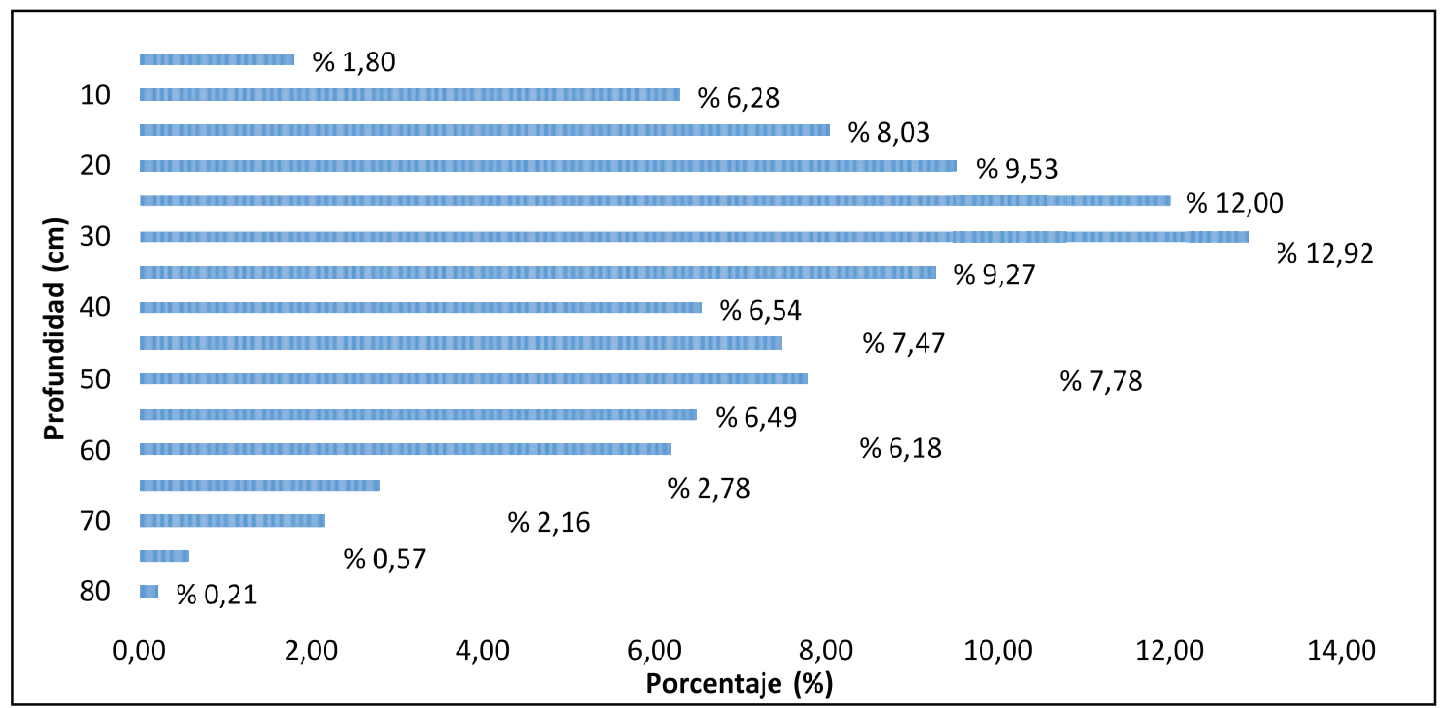

Figure 11

Patrón de extracción (\%) del tratamiento 3 (75\% agotamiento) a niveles de humedad del suelo en la papa (Solanum spp.) variedad victoria en función de la profundidad.

Table 2

Requerimiento hídrico y lámina de riego en el cultivo de papa (Solanum spp.) variedad victoria.

\begin{tabular}{|c|c|c|c|c|c|c|}
\hline \multirow[t]{2}{*}{ Etapa } & \multicolumn{2}{|c|}{ Tratamiento 1} & \multicolumn{2}{|c|}{ Tratamiento 2} & \multicolumn{2}{|c|}{ Tratamiento 3} \\
\hline & $\mathbf{R h}$ & Lr & $\mathbf{R h}$ & Lr & $\mathbf{R h}$ & Lr \\
\hline Inicial & 262,42 & 6,31 & 214,12 & 5,96 & 144,85 & 4,87 \\
\hline \multicolumn{2}{|c|}{ Desarrollo57,72 } & 2,88 & 43,46 & 2,73 & 80,4 & 2,98 \\
\hline \multicolumn{2}{|c|}{ Intermedic15,96 } & 3,03 & 12,96 & 2,14 & 9,37 & 2,57 \\
\hline Final & 152,46 & 4,07 & 184,74 & 4,26 & 193,81 & 4,31 \\
\hline Total & 488,56 & 16,29 & 455,28 & 15,09 & 428,43 & 14,73 \\
\hline
\end{tabular}

Rh: requerimiento hídrico $(\mathrm{mm})$; Lr: lámina de riego $(\mathrm{mm})$.

que al comprar con investigaciones [31, 42], indica valores de $409 \mathrm{~mm}$ y $416,7 \mathrm{~mm}$, considerar que la campaña en que se implementó el ensayo fue condicionante en la necesidad hídrica. Otros investigadores [31, 42] mencionan que el requerimiento de agua varía entre 350 y 600 mm, dependiendo del período de duración del cultivo, condiciones ambientales, tipo de suelo y variedad, además la producción disminuye si no se aporta con más del 50 por ciento del total de agua disponible en el suelo durante el crecimiento.

\subsection{Rendimiento por Categorías}

El análisis de varianza para el rendimiento categoría comercial, señala diferencias significativas entre tratamientos $\left(24,31^{*}\right)$, con un coeficiente de variación $10,64 \%$ y un 


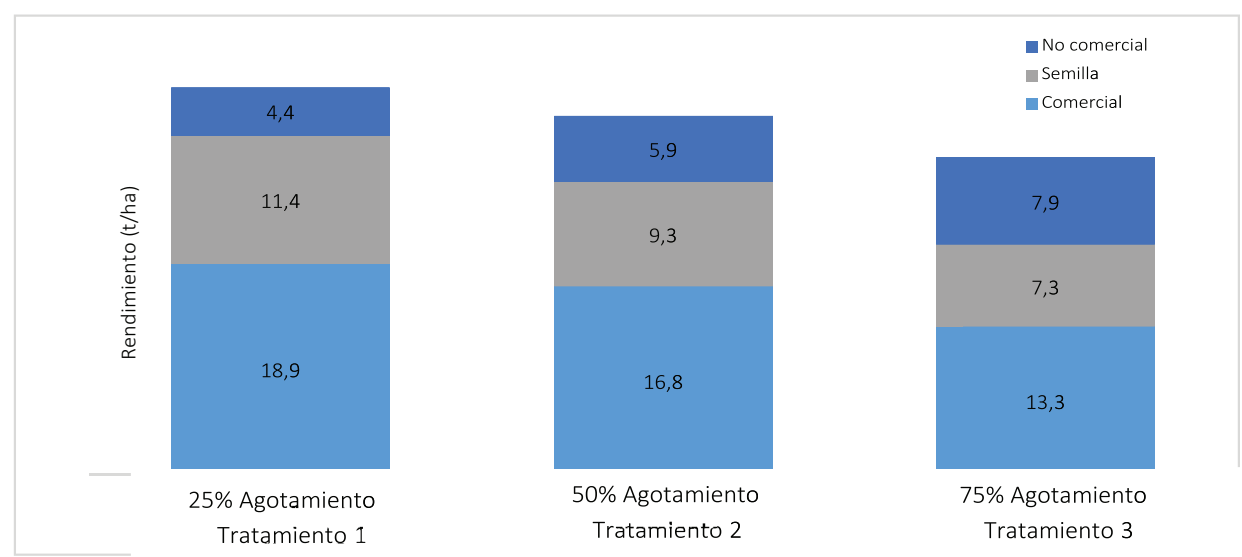

Figure 12

Promedios de rendimiento por tamaño del tubérculo (t/ha) a niveles de humedad del suelo en la papa (Solanum spp.) variedad victoria.

promedio general de 16,33 t/ha; por lo tanto el mayor rendimiento categoría 'comercial' fue para el tratamiento 1 con 18,89 t/ha y el menor rendimiento es el tratamiento 3 con 13,27 t/ha (Figura 11), con una diferencia del 5,62 t/ha, destacar que la escasez de agua reduce el tamaño de los tubérculos en 'calidad', es decir perdidas en el potencial de rendimiento [30, 32, 43]. Además de una malformación de tubérculos en forma de mancuerna o puntiagudos $[41,44]$ y según el avance el ciclo ocasiona defectos del tubérculo, como la mancha marrón interna, especialmente cuando el follaje está muerto y el suelo expuesto al sol [25].

En referencia al rendimiento de papa en categoría 'semilla', se obtuvieron diferencias significativas entre tratamientos $\left(12,75^{*}\right)$, con un coeficiente de variación de $13,5 \%$ y un promedio general de 9,33 t/ha; por lo tanto el tratamiento 1 presenta el mayor rendimiento con 11,4 t/ha y el tratamiento 3 presenta el menor con 7,3 t/ha (Figura 11), en tal sentido al no satisfacer con el requerimiento hídrico del cultivo, estos darán como resultados tubérculos de mala calidad, correlacionado con lo señalado por [45], indican que el déficit hídrico puede causar desórdenes en el crecimiento del tubérculo, brotación secundaria, tubérculos de tamaño más pequeño debido a la maduración alternada con recrecimiento de la cobertura foliar y la expansión celular cíclica de los tubérculos [38].

En el rendimiento de papa categoría 'no comercial', señala diferencias significativas entre tratamientos $[29,38]$, con un coeficiente de variación de 13,99\% y un promedio general de 6,09 t/ha; por lo tanto el tratamiento 3 presenta el mayor registro con 7,9 t/ha y el tratamiento 1 el menor con 4,4 t/ha (Figura 11), notando claramente que la calidad es preponderante, lo que concuerda con [18], el suministro adecuado de agua es determinante desde el inicio de la tuberización hasta que la planta alcance la madurez fisiológica para obtener altos rendimientos de 'excelente calidad', es decir al 
cumplir con el requerimiento hídrico del cultivo los resultados se verán reflejos en su rendimiento con la obtención de tubérculos de calidad.

\subsection{Rendimiento Total}

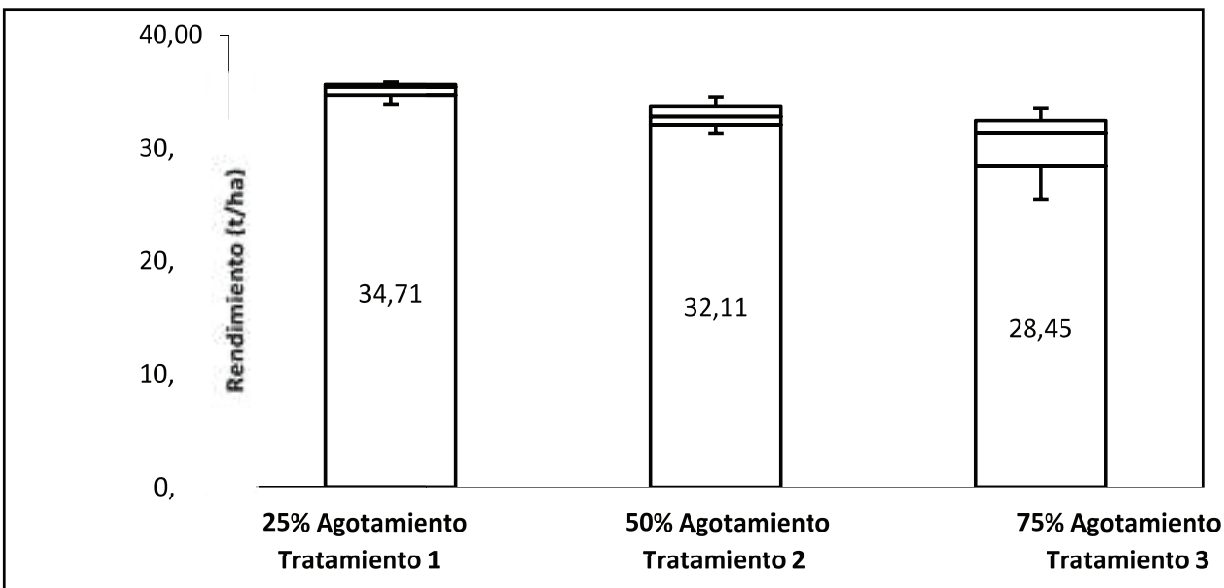

Figure 13

Promedios de rendimiento total (t/ha) a niveles de humedad del suelo en la papa (Solanum spp.) variedad victoria.

El análisis de varianza para el rendimiento total, señala diferencias no significativas para tratamientos $(29,73 \mathrm{~ns})$, con un coeficiente de variación $6,95 \%$ y un promedio general de 31,76 t/ha, esto es debido a que las categorías de papas representaron un solo peso (Figura 12), por su parte [12], señala que el contenido de humedad del suelo no debe presentar un umbral menor al $50 \%$ es decir mantener el suelo a capacidad de campo, donde el agua esté disponible para el cultivo sobre todo en etapas críticas, por el contrario se ve afectado el rendimiento del tubérculo directamente al restringir la transpiración y la fotosíntesis e indirectamente al reducir la evaporación del suelo y las hojas, con el aumento de la temperatura del suelo y de la planta [34, 39].

\section{Conclusiones}

El comportamiento radicular de la papa variedad victoria en función a la disponibilidad de agua en el suelo ocasionó que el tratamiento 3 con el 75\% de agotamiento desarrolle sus sistema radicular hasta los $78 \mathrm{~cm}$; seguido del tratamiento 2 con el $50 \%$ agotamiento hasta $67 \mathrm{~cm}$ y el tratamiento 1 con 25\% agotamiento hasta con $60 \mathrm{~cm}$, por lo tanto si la planta presenta poca disponibilidad de agua esta genera estrategias a través del crecimiento de su sistema radicular en la búsqueda de agua en capas más profundas del suelo. 
El cultivo de papa variedad victoria ante la aplicación de los tratamientos se estableció una relación requerimiento hídrico y rendimiento es así que el tratamiento 1 (25\% agotamiento) presentó un requerimiento de $488,46 \mathrm{~mm}$ con un rendimiento de 34,71 t/ha, el tratamiento 2 (50\% agotamiento) 455,28 $\mathrm{mm}$ con un rendimiento de 32,11 t/ha y el tratamiento 3 (75\% agotamiento) $428 \mathrm{~mm}$ con un rendimiento de $28,45 \mathrm{t} / \mathrm{h}$, por lo tanto niveles de restricción de agua superiores al 75\% generarían grandes pérdidas con bajos rendimientos debido a que en la zona el rendimiento promedio es de 35 t/ha.

La restricción de agua en el suelo ocasionó menor división celular lo que se reflejó en la parte aérea de la planta además de la reducción en la duración del ciclo de cultivo con 78,58 cm y 132 días para el tratamiento 1 (25\% agotamiento); 75,04 cm y 126 días para el tratamiento 2 (50\% agotamiento); $71,68 \mathrm{~cm}$ y 121 días para el tratamiento 3 (75\% agotamiento).

\section{Agradecimientos}

Se agradece al equipo técnico del Centro Experimental del Riego (CER), al Grupo de Investigación y Transferencia de Tecnología en Recursos Hídricos (GITRH) y a la Facultad de Recursos Naturales (FRN) de la ESPOCH.

\section{Conflicto de Intereses}

Los autores declaran no tener conflictos de intereses financieros ni personales que puedan influir inapropiadamente en el desarrollo de esta investigación.

\section{References}

[1] FAO. Statistical water [Base de datos FAOSTAT]. Roma: FAO; 2016.

[2] Farooq M. Función fisiológica de forma exógena aplicada glycinebetaine en la mejora de tolerancia a la sequía de la aromática de grano fino arroz. Agronomy Journal. 2008;194:325-333.

[3] FAO. New light on a hidden treasure. Roma: Estudio FAO Riego y Drenaje 56. Roma: FAO; 2009.

[4] Taylor H, Ratliff L. Root elongation rates of cotton and peanuts as a function of soil strength and soil water content. Soil Science. 1969;108:113-119.

[5] Thiele G, Theisen K, Bonierbale M, Walker T. Targeting the poor and hungry with potato science. Journal Potato.2010; 37:75-86. 
[6] Thompson A, Andrews J, Mulholland B, Mckee J, Hilton H, Horridge J. Overproduction of abscisic acid in tomato increases transpiration efficiency and root hydraulic conductivity and influences leaf expansion. P. Physiology. 2007;43:105-117.

[7] Van Loon CD. The effect of water stress on potato growth, development, and yield. Am. Potato J. 1981;58:51-69.

[8] Lynch JP. Turner review no, 14 roots of the second green revolution. Aust Journal. Bot. 2007;55:493-512.

[9] Hodge A. The plastic plant: Root responses to heterogeneous supplies of nutrients. New Phytol. 2004;162:9-24.

[10] Jaleel C. Estrés hídrico déficit efectos sobre el metabolismo de oxígeno reactivo en Catharanthus roseus. B: Biointerfaces. 2008;62:105-111.

[11] Jones A. Effect of soil texture on critical bulk densities for root growth. Soil Sci. Soc. Am. J. 1983;47:1208-1211.

[12] Kang S, Zhang J. Controlled alternate partial root-zone irrigation: Its physiological consequences and impact on water use efficiency. Journal. Exp. 2004;55:24372446.

[13] Onder S, Caliskan M, Onder D, Caliskan S. Different irrigation methods and water stress effects on potato yield and yield components. Agricultural Water Management. 2005;73:73-86.

[14] Santos T, Lopes C, Rodrigues M, De Souza C, Da-Silva J, Maroco J. Effects of deficit irrigation strategies on cluster microclimate for improving fruit composition of moscatel field-grown grapevines. Sci. hortic. 2007;112:321-330.

[15] Opena G, Porter G. Soil management and supplemental irrigation effects on potato: root growth. Journal Agronomy. 1999;91:426-431.

[16] Allen R, Pereira R, Raez D, Smith M. Estudio FAO Riego y Drenaje. Rome: FAO; 2006. Evapotranspiración de cultivo. Guías para la determinación de los requerimientos de agua de los cultivos.

[17] Cuesta X. INIAP - PNRT - papa. Vol 1. Quito: Planeta; 2008. Guía para el manejo y toma de datos de ensayos de mejoramiento de papa.

[18] Sermet, Caliskan, Caliska. Different irrigation methods and water stress effects on potato yield and yield components. Agricultural Water Management. 2005;73:73-86.

[19] Ahmadi S, Andersen M, Plauborg F, Poulsen R, Jensen C, Sepaskhah A. Effects of irrigation strategies and soils on field grown potatoes. Yield and water productivity. Agricultural Water Management. 2010;1:97- 109.

[20] Garay O. Uso consuntivo de agua de los cultivos. Journal Agronomy. 2009;2:9-15.

[21] Alva A, Moore A, Collins H. Impact of deficit irrigation on tuber yield and quality of potato cultivars. Crop Improvement Journal. 2012;26:211-227.

[22] Beltri E. Medida del balance hídrico y estimación del coeficiente Kc para la mejora de la programación de riegos. Técnicas de riego deficitario controlado. 2008. 
[23] FAO. Estudio FAO Alimento y Agua. Vol 10. Roma: FAO; 2008. El cambio climático y la producción de alimentos.

[24] Simpfendofer. Efecto del riego en el cultivo de papa: riego y drenaje. INIA. 2000;34:35-38.

[25] Haverkort, A. Water management in potato production. Technical Information Bulletin, International Potato Center (CIP). 1982;15:22.

[26] Deblonde P, Ledent J. Effects of moderate drought conditions on green leaf number, stem height, leaf length and tuber yield of potato cultivars. Agronomy Journal. 2001;14:31-41.

[27] Bengough A, Mullins C. The resistance experienced by roots growing in a pressurised cell-a reappraisal. Plant Soil. 1990;123:73-82.

[28] Dechassa N, Schenk M, Claassen N, Steingrobe B. Phosphorus Efficiency of Cabbage (Brassica oleraceae L. var. capitata), Carrot (Daucus carota L.), and Potato (Solanum tuberosum L.). Plant and Soil. 2003;250:215-224.

[29] Dexter A. Soil physical quality. Part I. Theory, effects of soil texture, density, and organic matter and effects on root growth. Geoderma. 2004;120:201-214.

[30] Benjamin J, Nielsen D. Water deficit effects on root distribution of soybean, field pea and chickpea. Field Crops Res. 2006;97:248-253.

[31] Songsri, P., Jogloy, S., Vorasoot, N., Akkasaeng, C., Patanothai, A., Holbrook, C. Root distribution of drought-resistant peanut genotypes in response to drought. Journal of Agronomy and Crop Science. 2008;194:92-103.

[32] Joshi M, Fogelman E, Belausov E, Ginzberg, I. Potato root system development and factors that determine its architecture. Agricultural Research Organization. 2016;

[33] Stalham M, Allen E. Water uptake in the potato (solanum tuberosum) crop. Journal Agricultural. 2004;142:373-393.

[34] Vasconcelos A, Casagrande D, Perecin L, Jorge E, Landell. Evaluación del sistema radicular de la caña de azúcar por diferentes métodos. Journal Root. 2003;12:56 75.

[35] Zhang J, Davies W. Abscisic acid produced in dehydrating roots may enable the plant to measure water status of the soil. Plant, cell and environment. Journal Biotechnology. 1989;5:73-81.

[36] Muñoz F, Mylavarapu R, Hutchinson C, Portier K. Root Distribution under seepageirrigated potatoes in northeast Florida. American Journal Potato. 2005;83:463-472.

[37] Ragassi C, Favarin J, Shiraishi F, Moita A, Henry S, De Melo P. Efeito da descompactacão profunda de solo na prod. Ao da cultura da batata. 2009;27:484489.

[38] Coelho E, OR D. Root distribution and water uptake patterns of corn under surface and subsurface drip irrigation. Plant and Soil. 1999;206:123-136. 
[39] Wang F, Kang Y, Liu S. (2006). Effects of drip irrigation frequency on soil wetting pattern and potato growth in North China Plain. Agricultural Water Management. 2006;79:248-264.

[40] Mingo D, Theobald J, Bacon M, Davies W, Dodd I. Biomass allocation in tomato (Lycopersicon esculentum) plants grown under partial rootzone drying: enhancement of root growth. Functional Plant Biology. 2004;31,971-978.

[41] Martin D, Stegman E, Fereres E. Management of Farm Irrigation Systems. Hoffman GJ, Howell TA, Solomon KH, editors. American Society of Agricultural Engineers; (1990). Irrigation scheduling principles.

[42] Sood M, Singh N. (2003). The potato: Production and utilization in sub-tropics. Journal Water Management.2003;32:121 - 128.

[43] Charkowski A. (2012). Cultivos de hortalizas. Plant Path. Consultado el 15 de febrero de 2018. Available from http://www.plantpath.wisc.edu. Wisconsin-Madison.

[44] Mackerron D, Jefferies R. The distributions of tuber sizes in droughte and irrigated crops of potato I. Observations on the effect of water stress graded yields from different cultivars. Potato Research. 1988;31:269-278.

[45] Jensen C, Jacobsen S, Andersen $M$ et al. Leaf gas exchange and water relation characteristics of field quinoa (Chenopodium quinoa Willd.) during soil drying. European Journal Agronomy. 2000.

[46] Peña R. (2014). Evaluación de seis genotipos de papa con tolerancia al déficit hídrico Chimborazo Ecuador. VI Congreso Ecuatoriano de la Papa; 2014 Jul 8-12. Ibarra: Brown; 2015. p. 190 - 194. 